\title{
A Tabletop System Using Infrared Image Recognition for Multi-user Identification
}

\author{
Shota Suto $^{1}$ and Susumu Shibusawa ${ }^{2}$ \\ ${ }^{1}$ Ibaraki University, Graduate School of Science and Engineering, Nakanarusawa 4-12-1, \\ Hitachi, Ibaraki 316-8511, Japan \\ 12nd3 04x@hcs.ibaraki.ac.jp \\ ${ }^{2}$ Ibaraki University, Dept. of Computer and Information Sciences, Nakanarusawa 4-12-1, \\ Hitachi, Ibaraki 316-8511, Japan \\ sibusawa@mx.ibaraki.ac.jp
}

\begin{abstract}
Many tabletop systems have been developed to facilitate face-to-face collaboration and work at small meetings. These systems often require users to attach sensors to their bodies to identify their positions, but attaching a sensor to one's body can be bothersome and annoying, and user position and posture may be restricted depending on where the sensor is attached. We have proposed a technique for estimating user position in a tabletop system by image recognition and implemented a tabletop system having a user position identification function incorporating the proposed technique. This technique first obtains touch points and hand-area information from touch operations performed by the user, and establishes an association between the touch points and hand from those positional relationships. Since the direction in which a hand is extended can be derived from that hand's touch information, the position of the user of the touch points belonging to that hand can be estimated. As part of this study, we also implemented a photo-object manipulation application, which has a function for orienting a photo object to face the user based on the results of the above user-position estimation technique. We performed an experiment to evaluate the position identification rate, and found that the proposed technique could identify user position with high accuracy.
\end{abstract}

Keywords: Tabletop system, Image recognition, FTIR, Multi-touch, User position identification, Area extraction.

\section{$1 \quad$ Introduction}

A tabletop system facilitates face-to-face collaboration at small meetings or any setting where people come together to work as a group. It enables all users to manipulate or examine displayed information and enables a variety of input operations to be executed by touching displayed information and performing gestures. Research surrounding tabletop systems with these features has been active including studies of interactive techniques [1], [2], development of information display technologies [3], [4], and the use of tabletop systems for supporting collaborative work [5], [6], [7]. 
In a tabletop system, objects can be oriented in various ways by touch gestures performed by multiple users, which means that a user may find it difficult to understand the text or photo of an object that is currently not facing in the user's direction. In response to this problem, several techniques have been developed to identify user positions by attaching sensors to chairs or the users themselves [8], [9], [10], and these techniques are used to automatically modify the orientation of objects according to the position of the user manipulating the objects. For example, Diamond Touch [9], registers beforehand each user and the user's position by having each user sit on a conductive sheet for user-identification purposes, and uses this information to determine the position of the user whenever the user is identified. However, identifying user position by attaching sensors to chairs or people can be troublesome, and time must be devoted to learning how to use sensor equipment. User posture may also be restricted depending on where the sensor is attached.

On the other hand, research has been performed on an interactive system that extracts images of body extremities using image recognition technology so that physical movements performed by the user can be used as input operations [11]. This kind of interactive system using image recognition negates the need for wearing a sensor thereby enabling users to use the system in a free and natural manner.

We propose a technique for estimating user position by image recognition in a tabletop system and construct a tabletop system incorporating this technique. This technique negates the need for wearing a sensor and removes restrictions depending on where the sensor is attached. This system uses a frustrated total internal reflection (FTIR) multi-touch panel and obtains touch points and hand-area information from user touch operations using image recognition. It establishes an association between the touch points and hand from those positional relationships. Since the direction in which a hand is extended can be derived from that hand's area information, the position of the user of the touch points belonging to that hand can be estimated. This study includes the implementation of a photo-object manipulation application that enables users to manipulate photo objects on the tabletop system by touch operations. The application has a function for orientating a photo object to face the user according to the results of estimating user position from touch gestures.

\section{Proposed System}

\subsection{System Configuration}

For this study, we designed a tabletop system with a multi-touch panel capable of touch-point recognition by the FTIR method [12]. This system features an infrared Web camera installed beneath the table to capture infrared images on the tabletop by picking up the acrylic panel on the table via a mirror. The system also includes a projector connected to a personal computer (PC) to display photo objects to users by projecting images onto the acrylic panel from underneath the table. In addition, tracing paper is pasted onto the acrylic panel to act as a screen and users are presented with information by having the projector project images onto this tracing paper. System configuration is shown in Figure 1. 
Finally, to obtain information on hand area, an infrared light is installed on the ceiling above the table. Since a hand on the tabletop will block infrared beams emitted from this light, an infrared shadow corresponding to the hand will form. The Web camera is used to pick up this shadow effect.

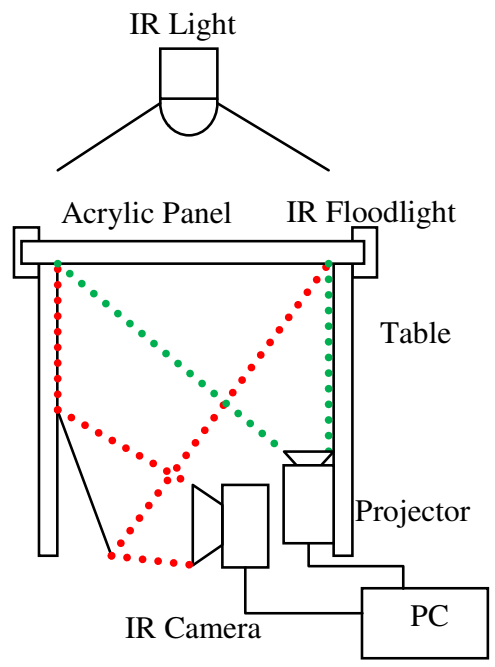

Fig. 1. System configuration

\subsection{Overview of User-Position Estimation Technique}

In a tabletop system, a user typically extends a hand from the edge of the table to manipulate some object. Accordingly, if it were possible to determine the direction from which the hand including touch points is being extended, the position of the user generating those touch points could be estimated.

In FTIR-based touch-point recognition as used here, the system picks up touch points as white light owing to the diffused reflection of infrared light. It also picks up the area occupied by a hand as a shadow generated by the blocking of light emitted from the infrared light installed on the ceiling. At this time, the system uses brightness values in the captured image to differentiate from the background image in two ways. Specifically, it extracts touch areas having a higher brightness value than the background and an area having a change in brightness value as a hand area. This extracted hand area includes the touch areas as a subset. Thus, when focusing on certain touch points, a hand area that includes those touch areas as a subset certainly exists, which means that the direction from which that hand is being extended can be determined from that hand-area information. As a result, the position of the user associated with certain touch points can be estimated since touch points and user position can be indirectly connected through the direction of extension of the hand to which those touch points belong. Extraction of these key areas and estimation of user position are outlined in Figure 2. 


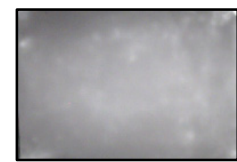

Background image

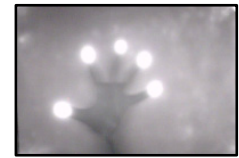

Captured image
Extract portions with high brightness

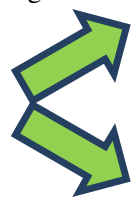

Extract portion having a change in brightness value

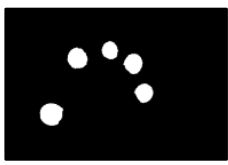

Extract touch areas

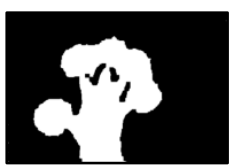

Extract hand area

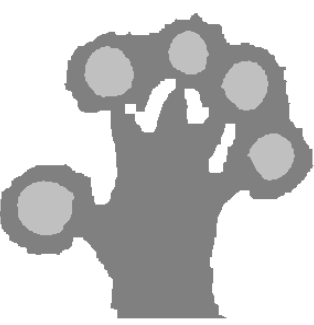

Judge that these touch operations are performed by the user positioned in the downward direction

Fig. 2. Extraction of key areas and estimation of user position

\subsection{User-Position Estimation Model}

The FTIR touch-point recognition system picks up touch points as white light and the hand area as a shadow of infrared light. Establishing a difference with the background image enables the touch areas and the hand area that includes those touch areas to be extracted. An image of a touch area and that of a hand area extracted by background differentiation are shown in Figures 3 (a) and (b), respectively.

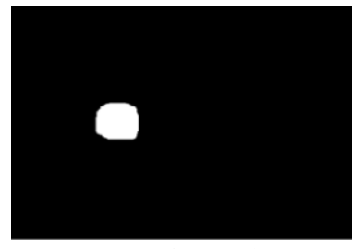

(a) Image of a touch area

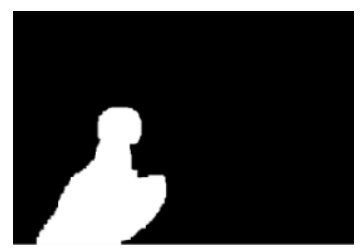

(b) Image of a hand area

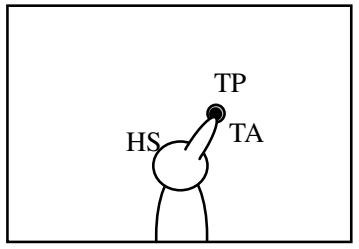

(c) Positional relationship among, $T A, T P$, and $H S$

Fig. 3. Extraction of touch-point and hand areas and their positional relationship

The positional relationship among touch area $(T A)$, touch point $(T P)$, and hand shadow $(H S)$ is shown in Figure $3(\mathrm{c})$, where the outer circle and inner circle at the fingertip of $H S$ corresponds to $T A$ and $T P$, respectively. This technique determines $T P$ by calculating the center of gravity of $T A$. When we define hand-area Hand as the union of $T A$ and $H S, T P$ is an element of Hand.

The proposed technique first investigates the attribution relation between $T P$ and Hand. It next determines from which edge in the image the Hand to which TP belongs to is extending. It finally estimates user position by drawing a correspondence between that direction and the touch point. 


\subsection{User-Position Estimation Technique}

More than one hand area may exist on the tabletop at any one time. To recognize individual hand areas, the technique labels areas having connected pixels and assigns the label $L$ to each hand area.

Then, when focusing on a certain touch point $T P$, that a hand area Hand that includes the coordinates of $T P$ as an element exists. The results of labeling can therefore be used to extract label $L[T P(x, y)]$ corresponding to the coordinates of $T P$. Here, $L[T P(x, y)]$ is equivalent to label $L[H a n d]$ including $T P$. The hand area corresponding to the targeted touch point can therefore be identified by referring to $\operatorname{LiTP}(x, y)]$.

Referring to Figure 3(b), it can be seen that Hand consists of a continuous area connected to a certain edge of the image. Accordingly, if that edge can be determined, the direction from which Hand is being extended can likewise be determined.

\section{System Implementation}

Our prototype tabletop system has a height of $70 \mathrm{~cm}$, a panel $100 \mathrm{~cm} \times 90 \mathrm{~cm}$ in size, and a display manipulation range of $60 \mathrm{~cm} \times 50 \mathrm{~cm}$. The tabletop is shown in Figure 4 .

We also implemented an application for manipulating photo objects by touch gestures. This application reads in image data as photo objects and displays them on the tabletop system. It treats touch gestures made by the user as input and generates results in response to those gestures. Touch gestures are listed and described in Table 1. Change direction is an operation which makes the object face the user when touching it with three fingers. An example of the change-direction gesture is shown in Figure 5.

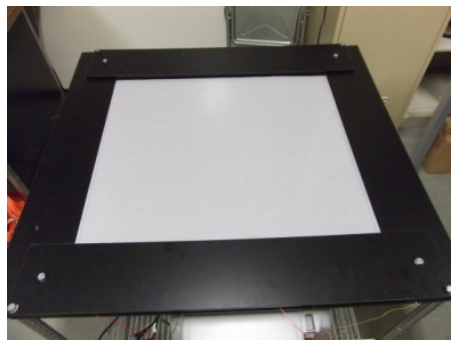

Fig. 4. View of tabletop

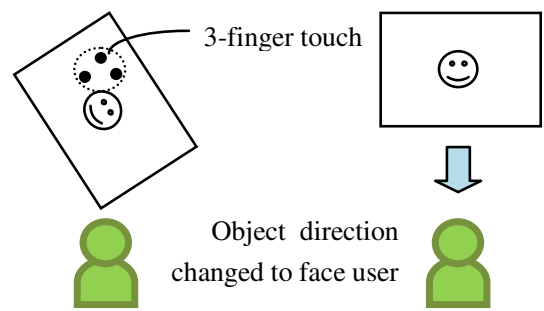

Fig. 5. Change-direction gesture

Table 1. Touch gestures

\begin{tabular}{|l|c|l|}
\hline \multicolumn{1}{|c|}{ Operation } & No. of Touches & Description \\
\hline Move & 1 & Move object \\
\hline Zoom in/out & 2 & Change object size \\
\cline { 3 - 3 } Rotate & & Rotate object \\
\hline Change direction & 3 & Change object's direction to face user \\
\hline
\end{tabular}




\section{Evaluation Experiment}

\subsection{User-Identification Evaluation Experiment}

To assess the accuracy of the user-position estimation technique, we conducted a subjective experiment using the 3-finger change-direction gesture. After briefing four subjects on how to perform this gesture, we asked each to perform the gesture ten times on the tabletop system in each of the up, down, left, and right directions. At this time, we recorded actual user position and system-estimated position in order and determined position identification rate by comparing the values obtained. Specifically, denoting the number of times this gesture was performed as $d_{a c t}$ and the number of times that actual user position agreed with system-estimated position as $d_{\text {correct }}$, we computed position identification rate by Eq. (1).

$$
\text { position identification rate }=\frac{d_{\text {correct }}}{d_{\text {act }}} \times 100[\%] \text {. }
$$

The experiment was performed in the evening after sunset considering the possible effects of infrared light contained in sunlight. The prototype tabletop was installed in the center of a room and two infrared lights were installed on the ceiling above the table. Each light was $90-\mathrm{cm}$ long incorporating six equally spaced infrared LEDs and the two lights were set $70 \mathrm{~cm}$ apart. The distance from the ceiling to the tabletop panel was $185 \mathrm{~cm}$.

\subsection{Results and Discussion}

Average identification rate for the change-direction gesture by four subjects for each of the four tabletop directions and overall average for all directions are shown in Figure 6.

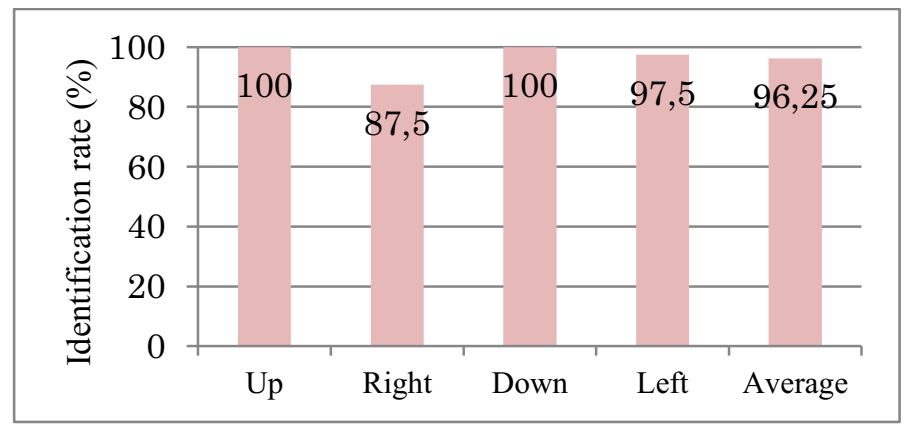

Fig. 6. Identification rate for change-direction gesture

Average identification rate for the change-direction gesture was found to be about $96 \%$. Examining these results, it can be seen that hand extension from the up and down tabletop directions could be accurately identified. There were times, however, when hand extension from the left and right tabletop directions could not be accurately 
identified. Examples of incorrect hand-extension identification from the left and right directions are shown in Figure 7. Images for a change-direction gesture from the left are shown in Figures 7 (a) and (b) and those for a change-direction gesture from the right are shown in Figures 7 (c) and (d).

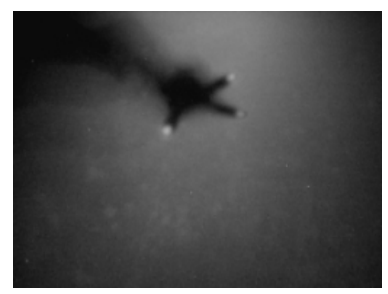

(a) Captured image

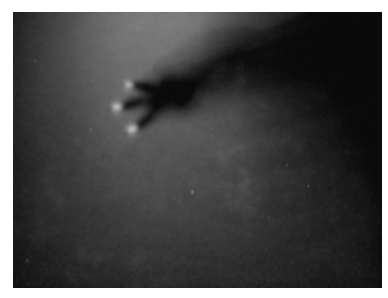

(c) Captured image

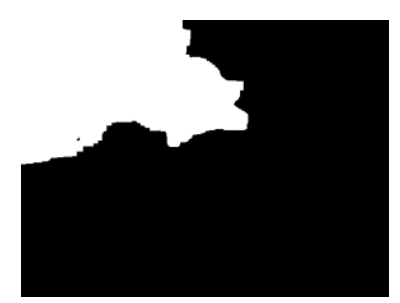

(b) Hand area extracted

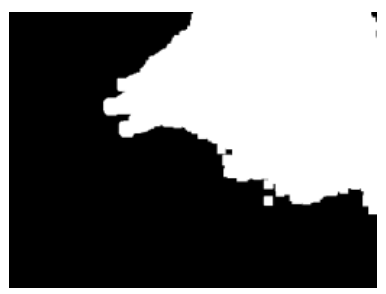

(d) Hand area extracted

Fig. 7. Change-direction gesture from left and right directions

In the examples of Figure 7, the number of pixels in the hand shadow area crossing an edge are higher in the up direction than in the left and right directions. As a result, the system erroneously judges those tabletop operations to be those of a user positioned at the top of the tabletop (in the up direction). This is because the touch-point coordinates on the captured image cannot necessarily be used to correctly obtain the touch-point coordinates on the acrylic panel.

\section{Conclusion}

We proposed a technique for identifying user position in a tabletop system through image recognition and introduced a prototype tabletop system incorporating this technique. We also implemented a photo-object manipulation application for running on the tabletop and conducted a subjective experiment to evaluate the accuracy of the proposed technique in estimating user position. In this experiment, we recorded actual user position and system-estimated position when subjects made change-direction gestures and calculated the position identification rate by determining the rate of agreement between actual and estimated positions. We found that an average position identification rate of about $96 \%$ could be achieved in the case of change-direction gestures, which demonstrates that user position can be estimated by the proposed image 
recognition technique. The technique is a simple technique for identifying the user position and negates the need for wearing a sensor.

Errors in identifying user position occurred in the system as a result of camera image compensation. An offset between the manipulation area and display area on the tabletop prevented the system from correctly recognizing how a hand shadow area was crossing an edge making it easy for erroneous judgments to occur. We considered that using a wide-angle camera would be able to capture the entire tabletop panel head-on, which should eliminate complicated image-compensation processing by the system and suppress this phenomenon. In future research, we plan to evaluate the position identification rate during simultaneous operations by multiple users and to add gesture functions making full use of user position identification. We also look to apply the proposed technique to face-to-face collaborative work systems and to evaluate its usability in such an application.

\section{References}

1. Clifton, P., Mazalek, P., Sanford, J., Rébola, C., Lee, S., Powell, N.: SketchTop: Design Collaboration on a Multi-touch Tabletop. In: TEI 2011, pp. 333-336 (2011)

2. Haller, M., Brandl, P., Leithinger, D., Leitner, J., Seifried, T., Billinghurst, M.: Shared Design Space: Sketching Ideas Using Digital Pens and a Large Augmented Tabletop Setup. In: Advances in Artificial Reality and Tele-Existence, pp. 185-196 (2006)

3. Yamaguchi, T., Nacenta, M., Sakurai, S., Itoh, Y., Kitamura, Y., Subramanian, S., Gutwin, C., Kishino, F.: An Evaluation of Perspective Correction Regarding Locations of a User and Displays. IEICE Trans. on Information and System J91-D(12), 2746-2754 (2008)

4. Sakurai, S., Kitamura, Y., Subramanian, S.: A Tabletop Display with Visibility Control Using Revolving Polarizer. IPSJ Journal 50(1), 332-343 (2009)

5. Funamoto, S., Sugiyama, K.: Support and Evaluation for Group Discussions in Seminar Type Lectures Using Table Top Interface. In: Proc. of the 6th Knowledge Creation Support Systems Symposium, pp. 119-126 (2009)

6. Ohashi, M., Itou, J., Munemori, J., Matsushita, M., Matsuda, M.: Development and Application of Idea Generation Support System Using Table-Top Interface. IPSJ Journal 49(1), 105-115 (2008)

7. Goh, W.-B., Shou, W., Tan, J., Jackson Lum, G.T.: Interaction Design Patterns for Multi-touch Tabletop Collaborative Games. In: CHI EA 2012, pp. 141-150 (2012)

8. Watanabe, K., Takeuchi, T., Inoue, T., Okada, K.: Face-to-Face Collaboration System that Identifies Individual User. IEICE Transactions J91-D(12), 2755-2764 (2008)

9. Dietz, P., Leigh, D.: DiamondTouch: A Multiuser Touch Technology. In: UIST 2001, pp. 219-226 (2001)

10. Annett, M., Grossman, T., Wigdor, D., Fitzmaurice, G.: Medusa: A Proximity-ware Multi-touch Tabletop. In: UIST 2011, pp. 337-346 (2011)

11. Mamiya, H., Sato, T., Fukuchi, K., Koike, H.: Tabletop Entertainment System with a Finger Opening and Closing Gesture. In: WISS 2007 (2007)

12. Han, J.Y.: Low-cost Multi-touch Sensing Through Frustrated Total Internal Reflection. In: UIST 2005, pp. 115-118 (2005) 